\title{
Desarrollo de un Manipulador Robótico de Cinco Grados de Libertad para fines de Investigación
}

Developing of a Robotic Manipulator of Five Degrees of Freedom for Research Purposes

Ernesto Díaz Ronceros ${ }^{1}$

\section{RESUMEN}

Objetivo: Diseñar, modelar e implementar una plataforma de investigación móvil basada en un robot manipulador de cinco grados de libertad. Métodos: Tipo de investigación: Tecnológica. La población está conformada por todas las plataformas de investigación robóticas. La muestra pertenece al tipo manipulador robótico de cinco grados de libertad. Como instrumento para la recolección de datos se utilizó la tabla de parámetros de Denavit-Hartenberg. Resultados: Se logró la implementación de la parte mecánica del manipulador, siendo esta liviana y estable. La tarjeta controladora de servomotores que se construyó para enlazar el ordenador al manipulador cumplió con la tarea encomendada. También se diseñó y modelo satisfactoriamente la interfaz en LabView para el control y asignación de coordenadas del manipulador. Conclusiones: Se cumplió con los objetivos, como: diseñar, modelar e implementar el manipulador robótico de cinco grados de libertad, con el fin de contar con una plataforma de investigación orientada al campo de la robótica en la etapa universitaria.

Palabras clave: Robot manipulador, Denavit Hartenberg, LabView, SolidWorks.

\section{ABSTRACT}

Objective: Design, modeling and implementation a mobile research platform based in a robot manipulator of five degrees of freedom. Methods: Type of investigation: technological. The population is formed for all robotics research platforms. The sample belongs to the type robotic manipulator of five degrees of freedom. As instrument for recollection of data used the Denavir Hatenberg parameters table. Results: The implementation was achieved of the mechanic part of manipulator, resulting light and stable. The controller card of servomotors that was constructed for link the ordenator with the manipulator, fulfilled with task entrusted. Also was designed and modeled satisfactorily the interface in LabView for the control and assignation of coordinates of the manipulator. Conclusions: The objectives as: design, model and implement the robot manipulator of five degrees of freedom, with the purpose of have a research platform oriented at the field of robotic at the university stage.

Keywords: Manipulator robot, Denavit Hartenberg, LabView, SolidWorks. 


\section{INTRODUCCIÓN}

Sabemos que hoy en día las investigaciones sobre la robótica se encuentran en una constante evolución y avance tecnológico, lo cual nos motiva a seguir desarrollando nuestras habilidades sobre como diseñar, modelar e implementar plataformas robóticas móviles; con el fin de llegar a conocer, experimentar y solucionar los problemas con respecto a temas como la cinemática directa, la cinemática inversa y la dinámica. Los robots manipuladores son un ejemplo de ello, Kelly (2003) menciona: "Un robot manipulador es una máquina manipuladora con varios grados de libertad controlada automáticamente, reprogramable y de múltiples usos, pudiendo estar en un lugar fijo o móvil para su empleo en aplicaciones industriales". Algunas de estas aplicaciones son: soldar, realizar ensamblajes, para levantar cargas pesadas, etc. $Y$ también algunos prototipos son orientados a los estudios de pre grado, en el cual los estudiantes pueden conocer los principios básicos de la robótica de forma experimental para luego ellos logren desarrollar prototipos propios e investigaciones de mayor envergadura y de impacto científico y social.

Estos manipuladores están formados por vínculos. Craig (2006). Un manipulador puede considerarse como un conjunto de cuerpos conectados en una cadena mediante articulaciones. Estos cuerpos se llaman vínculos o segmentos. Las articulaciones forman una conexión entre un par adyacente de vínculos. Barrientos, Peñín, Balaguer y Aracil (1997) indicaron: "Mecánicamente, un robot está formado por una serie de elementos o eslabones unidos mediante articulación que permiten un movimiento relativo entre cada dos eslabones consecutivos. El movimiento de cada articulación puede ser de desplazamiento, de giro, o de una combinación de ambos"

Para la generación de movimiento del robot se necesitan actuadores, los cuales. Barrientos et al. (1997) los define: Los actuadores tiene por misión generar el movimiento de los elementos del robot según órdenes dadas por la unidad de control. Los actuadores utilizados en robótica pueden emplear energía neumática hidráulica o eléctrica. Para realizar los movimientos del robot, este debe ser controlado mediante un sistema de comunicación. Bolton (2006) expresó: La tendencia actual de sistemas de comunicación omnipresentes, obliga al docente y al estudiante de ingeniería a involucrarse en estas tecnologías. Existen diversas tecnologías de comunicación como por ejemplo, Radio Frecuencia, Infrarrojo, Bluetooth, I2C, Ethernet, Serial.

Finalmente un manipulador robótico debe tener una posición en el espacio, Subir (2008). El movimiento de un cuerpo rígido en el espacio cartesiano tridimensional comprende la traslación y la rotación. Mientras que la traslación se define mediante el uso de las tres coordenadas cartesianas, la rotación necesita tres coordenadas angulares. En el estudio de la cinemática de manipuladores robóticos, se trata constantemente con la posición y orientación de varios cuerpos en el espacio. Los cuerpos de interés incluyen los eslabones del manipulador, las herramientas y las piezas de trabajo.

La justificación del presente trabajo se basa en la necesidad de contar con una plataforma de investigación orientada al campo de la robótica, específicamente un robot manipulador de cinco grados de libertad, para realizar estudios sobre la cinemática directa y la cinemática inversa; con esto también se busca motivar al estudiante a que cada uno logre desarrollar un prototipo, tomando como modelo y referencia el presente artículo. De esta manera logramos una mejor enseñanza - aprendizaje, transportando todo lo aprendido en la teoría a la práctica

Por lo tanto nuestra hipótesis será: Una plataforma de investigación basada en un robot manipulador de cinco grados de libertad nos permite realizar cálculos tanto de la cinemática directa como la inversa en tiempo real y visualizado en la interfaz diseñada en LabView.

\section{MATERIAL Y MÉTODOS}

La investigación corresponde a un estudio de tipo aplicativo y tecnológico porque nos permite utilizar este prototipo para realizar estudios de investigación relacionados a la robótica. La 
población estuvo comprendida por todas las plataformas de investigación robóticas que existen como pueden ser robots manipuladores, cuadrúpedos, hexápodos, omnidireccionales, etc. De los cuales nuestra muestra es la Plataforma de investigación de tipo robot manipulador.

\section{Instrumentos para la recolección de datos}

Tabla de Parámetros D-H: La tabla de Denavit - Hartenberg se realiza asignando sistemas de referencia para encontrar los valores de giro del eje $z$, translación en $z$, translación en $x$ y rotación en $x$. Utilizados para enlazar los eslabones del sistema. Barrientos et al. (1997) describe el procedimiento para la elaboración de la tabla es la siguiente:

a. Identificación de los Sistemas de Coordenadas Asociadas a cada Articulación

- Identificar del eje de desplazamientos de cada articulación.

- Identificar del origen de cada sistema de coordenadas.

- Identificar de los ejes z de cada sistema de coordenadas.

- Identificar de los ejes $x$ de cada sistema de coordenadas.

\section{b. Obtención de los 4 Parámetros de DH (q,a,,)}

Tabla 1. Parámetros Denavit - Hartenberg

\begin{tabular}{|c|c|c|c|c|}
\hline$i$ & $a_{i-1}$ & $\boldsymbol{a}_{\mathrm{i}-1}$ & & \\
\hline $\begin{array}{l}i+1 \\
i+2\end{array}$ & $\begin{array}{l}a_{i} \\
a_{i+1}\end{array}$ & $\begin{array}{l}a_{\mathrm{i}} \\
a_{\mathrm{i}+1}\end{array}$ & $\begin{array}{l}d_{i+1} \\
d_{i+2}\end{array}$ & $\begin{array}{l}q_{+1} \\
q_{+2}\end{array}$ \\
\hline
\end{tabular}

Dónde:

- Vínculo:面

- Ángulo de torsión: $\mathrm{a}_{\mathrm{i}-1}$

- Distancia de vínculo: $a_{\mathrm{i}-1}$

- Desplazamiento de vínculo: $d_{\mathrm{i}}$

- Ángulo de articulación: q

\section{Tabla de Requerimientos para Hardware de} Control

Para el hardware de control se necesita implementar una tarjeta servocontroladora, que permita recepcionar los datos enviados desde el ordenador para la posición de los servomecanismos.

Tabla 2. Requerimientos para Hardware de Control

\begin{tabular}{cc}
\hline Caracteristicas & Servo - Controladora \\
\hline PWM & 5 \\
Serial & 1 \\
Vsource & {$[4,5-9] \mathrm{V}$} \\
Interrupts & CCP1,RCIE \\
\hline
\end{tabular}

Diseño, Modelado E Implementación Del Robot Manipulador De Cinco Grados De Libertad.

\section{Análisis Cinemático del Manipulador}

Nuestro análisis cinemático se realizó a un manipulador de 5 grados de libertad, del cual se busca obtener la posición del efector final en función de los ángulos de las articulaciones $\left(\mathrm{q}_{1}, \mathrm{q}_{2}, \mathrm{q}_{3}\right)$ que están representadas con servomotores.

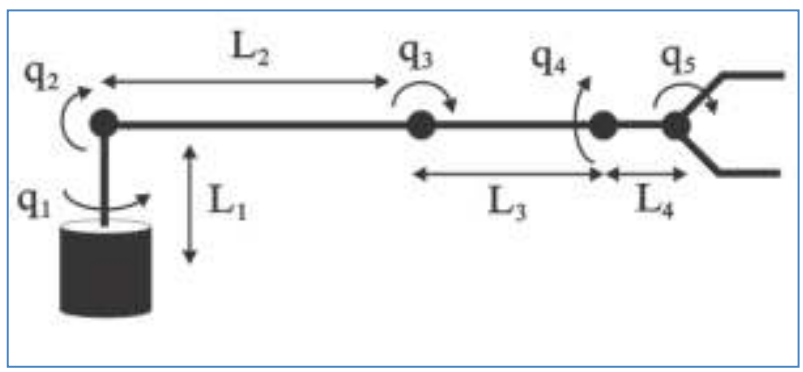

Figura 1. Cadena cinemática simplificada

\section{Cinemática Directa del Manipulador}

Se busca obtener los parámetros de Denavit Hartenberg, asignando sistemas de referencias a las articulaciones del manipulador como se muestra en la Figura 2 y posteriormente formular la tabla de parámetros de Denavit Hartenberg que se aprecia en la Tabla 3. Aplicando la regla de la mano derecha logramos obtener los sistemas de referencia del manipulador.

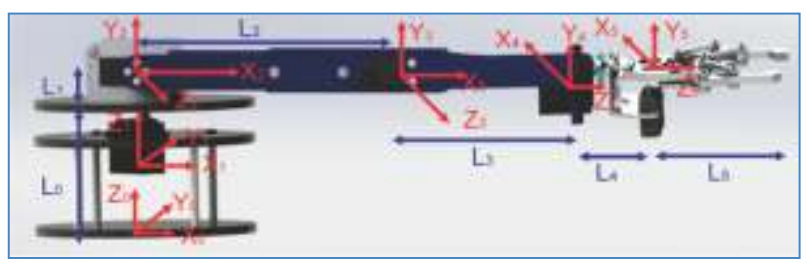

Figura 2. Sistema de referencia en el manipulador 
Tabla 3. Parámetros Denavit - Hartenberg para el manipulador

\begin{tabular}{ccccc}
\hline $\mathrm{i}$ & $\mathrm{a}_{\mathrm{i}-1}$ & $\boldsymbol{a}_{\mathrm{i}-1}$ & $\boldsymbol{d}_{\mathrm{i}}$ & $\mathrm{q}_{\mathrm{i}}$ \\
\hline 1 & 0 & 0 & $L_{0}$ & 0 \\
2 & 90 & 0 & $L_{i}$ & $\mathrm{q}_{1}$ \\
3 & 0 & $L_{2}$ & 0 & $\mathrm{q}_{2}$ \\
4 & -90 & $L_{3}$ & 0 & $\mathrm{q}_{3}$ \\
5 & 0 & $L_{4}$ & 0 & 0 \\
6 & 0 & $L_{5}$ & 0 & 0 \\
\hline
\end{tabular}

\section{Resolución de la Matriz de Transformación para Extremidades}

Las transformaciones:

${ }^{\mathrm{m} m \mathrm{~m} m \mathrm{~m} m \mathrm{~m}+1} A_{\mathrm{i}}=T\left(Z_{\mathrm{i}-1}, \mathrm{q}\right) \cdot T\left(0,0, d_{\mathrm{i}}\right) \cdot \mathrm{T}\left(a_{\mathrm{i}-1}, 0,0\right) \cdot \mathrm{T}\left(X_{\mathrm{i}} \mathrm{a}_{\mathrm{i}-1}\right)$

- Rotación alrededor del eje $Z_{\mathrm{i}-1 \square}$ un ángulo q

- Traslación a lo largo de $Z_{\mathrm{i}-1}$ una distancia $d_{\mathrm{i}}$; vector $d_{\mathrm{i}}\left(0,0, d_{\mathrm{i}}\right)$.

- Traslación a lo largo de $X_{\text {i }}$ una distancia $a_{\mathrm{i}-1}$; vector $a_{\mathrm{i}-1}\left(a_{\mathrm{i}-1}, 0,0\right)$.

- Rotación alrededor del eje $X_{\mathrm{i} \square}$ un ángulo $\mathrm{a}_{\mathrm{i}-1}$.

${ }^{\mathbb{M r} 1} A_{\mathrm{i}}=\operatorname{Rot}\left(Z_{\mathrm{i}-1}, \mathrm{q}_{\mathrm{i}}\right) \cdot \operatorname{Tras}\left(0,0, d_{\mathrm{i}}\right) \cdot \operatorname{Tras}\left(a_{\mathrm{i}-\mathrm{p}} 0,0\right) \cdot \operatorname{Rot}\left(X_{\mathrm{i}} \mathrm{a}_{\mathrm{i}-1}\right)$

Asumiendo:

$$
\begin{gathered}
\sin \mathrm{q}_{\mathrm{i}}=s \mathrm{q}_{\mathrm{i}} \\
\cos \mathrm{q}_{\mathrm{i}}=\mathrm{Fq} \\
\sin \mathrm{a}_{\mathrm{i}-1}=s \mathrm{a}_{\mathrm{i}-1} \\
\cos \mathrm{a}_{\mathrm{i}-1}=c \mathrm{a}_{\mathrm{i}-1}
\end{gathered}
$$

Obtenemos la multiplicación de las cuatro matrices:

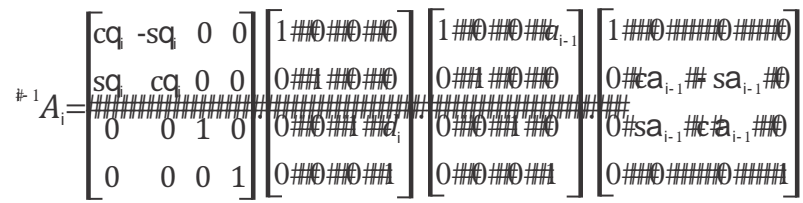

Resolviendo, obtenemos la Matriz de Transformación que relación los sistemas:

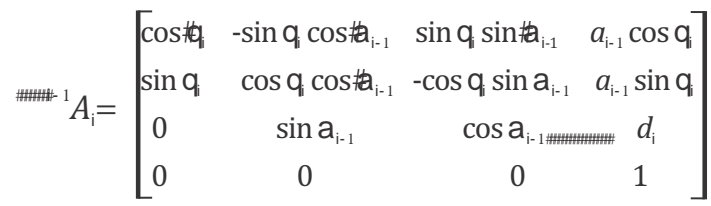

Desarrollando las Matrices para cada Sistemas:

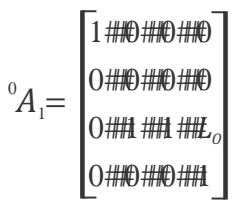

$$
A_{2}=\left[\begin{array}{cccc}
\cos q_{1} & 0 & \sin q_{1} & 0 \\
\sin q_{1} & 0 & -\cos q_{1} & 0 \\
0 & 1 & 0 & L_{1} \\
0 & 0 & 0 & 1
\end{array}\right]
$$

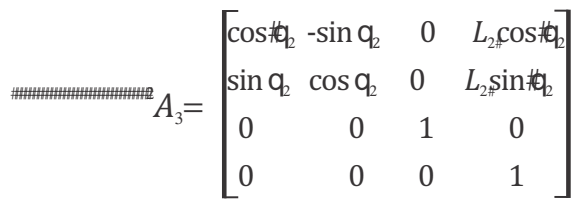

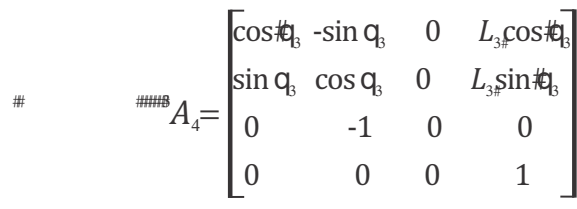

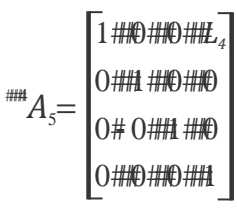

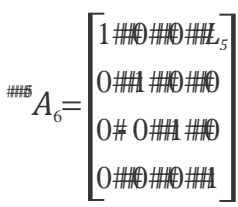

Matriz de Transformación final:

Para: $n=6$

$$
T={ }^{0} A_{n}={ }^{0} A_{1} \cdot{ }^{1} A_{2} \cdot{ }^{2} A_{3} \cdot{ }^{3} A_{4} \cdot{ }^{4} A_{5} \cdot{ }^{5} A_{6}
$$

Para fines abreviados se usará cq para representar $\cos \mathrm{q}_{\mathrm{i}}$ y $\mathrm{sq}_{\mathrm{i}}$ para $\sin \mathrm{q}_{\mathrm{i}}$ respectivamente.

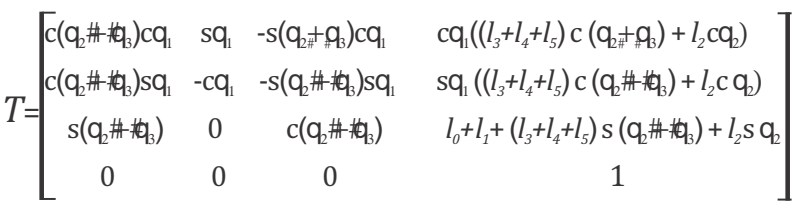


De la matriz obtenida, los elementos: $r 14, r 24$ y $r 34$; permiten describir la posición espacial en coordinadas cartesianas:

$x_{e}=\cos \mathrm{q}_{1}\left(\left(L_{3}+L_{4}+L_{5}\right) \cos \left(\mathrm{q}_{2}+\mathrm{q}_{3}\right)+L_{2} \cos \mathrm{q}_{2}\right)$

$y_{e}=\sin q_{1}\left(\left(L_{3}+L_{4}+L_{5}\right) \cos \left(q_{2}+q_{3}\right)+L_{2} \cos q_{2}\right)$

$\left.Z_{e}=L_{0}+L_{1}+\left(L_{3}+L_{4}+L_{5}\right) \sin \left(\mathrm{q}_{2}+\mathrm{q}_{3}\right)+L_{2} \sin \mathrm{q}_{2}\right)$

\section{Cinemática Inversa del Manipulador}

Luego de obtener las ecuaciones de la cinemática directa, ahora debemos hallar cuáles serán las ecuaciones de la cinemática inversa, que nos permita saber en qué posición se encuentran los ángulos de las articulaciones principales $q_{1},\left[q_{2}, q_{3}\right.$. También hay que tener en cuenta el sentido de giro de estos, si es negativo o positivo, de acuerdo a ello también variaran las ecuaciones.

Sea las ecuaciones:

$a=\left(L_{3}+L_{4}+L_{5}\right) \cos \left(q_{2}+q_{3}\right)+L_{2} \cos q_{2}$

$b=L_{0}+L_{1}+\left(L_{3}+L_{4}+L_{5}\right) \sin \left(\mathrm{q}_{2}+\mathrm{q}_{3}\right)+L_{2} \sin \mathrm{q}_{2}$

Reduciendo las ecuaciones:

$x_{e}=\operatorname{cosq}_{1}^{*} a$

$y_{e}=\sin _{1}^{*} a$

$z_{e}=b$

Dividiendo

$\frac{y_{e}}{x_{e}}=\operatorname{tanq}_{1}$

$\mathrm{q}_{1}=\tan ^{-1}(y e / x e)$

Operando las ecuaciones se deduce:

$a=\sqrt{x_{e}^{2}+y_{e}^{2}}$

$(a)^{2}+\left(b-L_{1}\right)^{2}=\left(\left(L_{3}+L_{4}+L_{5}\right) \cos \left(\mathrm{q}_{2}+\mathrm{q}_{3}\right)+L_{2} \cos \mathrm{q}_{2}\right)^{2}$ $\left.+\left(L_{3}+L_{4}+L_{5}\right) \sin \left(\mathrm{q}_{2}+\mathrm{q}_{3}\right)+L_{2} \sin \mathrm{q}_{2}\right)^{2}$

Agrupando y despejando a través de identidades trigonométricas:

$(a)^{2}+\left(b-\left(L_{0}-L_{2}\right)\right)^{2}=\left(L_{3}+L_{4}+L_{5}\right)^{2}\left(\cos ^{2}\left(\mathrm{q}_{2}+\mathrm{q}_{3}\right)+\sin ^{2}\left(\mathrm{q}_{2}+\mathrm{q}_{3}\right)\right)$ $+L_{2}^{2}\left(\cos ^{2} G_{q_{2}}+\sin ^{2} q_{2}\right)+2 L_{2}\left(L_{3}+L_{4}+L_{5}\right)$ $\left(\cos \left(\mathrm{q}_{2}+\mathrm{q}_{3}\right) * \cos \left[\Phi_{2}+\sin \left(\mathrm{q}_{2}+\mathrm{q}_{3}\right) * \sin \mathrm{q}_{2}\right)\right.$

$(a)^{2}+\left(b-\left(L_{0}-L_{1}\right)\right)^{2=}\left(L_{3}+L_{4}+L_{5}\right)^{2}+L_{2}^{2}+2 L_{2}\left(L_{3}+L_{4}+L_{5}\right) \cos q_{3}$ $\cos \mathrm{q}_{3}=\frac{(a)^{2}+\left(b-\left(L_{0}-L_{1}\right)\right)^{2}-L_{2}{ }^{2}-\left(L_{3}+L_{4}+L_{5}\right)^{2}}{2 L_{2}\left(L_{3}+L_{4}+L_{5}\right)}$

$\sin q_{3}=\sqrt{\sqrt{1}-\cos ^{2} q_{3}}, \tan q_{3}=\sin q_{3} / q_{3}$

$\theta_{3}=\cos ^{-}\left(\frac{x_{e}{ }^{2}+y_{e}{ }^{2}+z_{e}{ }^{2}+\left(L_{0}-L_{1}\right)^{2}-L_{2}{ }^{2}-\left(L_{3}+L_{4}+L_{5}\right)^{2}-2\left(L_{0^{-}}-L_{1}\right) z_{e}}{2 L_{2^{-}}\left(L_{3}+L_{4}+L_{5}\right)}\right)$

De las ecuaciones de a y $b$ :

$a^{*} \cos \mathrm{q}_{2}+\left(b-\left(L_{0}+L_{1}\right)\right) \sin \mathrm{q}_{2}=\left(L_{3}+L_{4}+L_{5}\right) \cos \left(\mathrm{q}_{2}+\mathrm{q}_{3}\right) * \cos \mathrm{q}_{2}$ $\left.\theta\left(L_{3}+L_{4}+L_{5}\right) \sin \left(\mathrm{q}_{2}+\mathrm{q}_{3}\right) * \sin \mathrm{q}_{2}\right)$

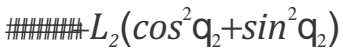

$a^{*} \cos \mathrm{q}_{2}+\left(b-\left(L_{0}+L_{1}\right)\right) \sin \mathrm{q}_{2}=\left(L_{3}+L_{4}+L_{5}\right)\left(\cos \left(\mathrm{q}_{2}+\mathrm{q}_{3}\right) * \cos \mathrm{q}_{2}\right.$ $\left.+\left(L_{3}+L_{4}+L_{5}\right) \sin \left(\mathrm{q}_{2}+\mathrm{q}_{3}\right) * \sin \mathrm{q}_{2}\right)+L_{2}$

$a^{*} \cos q_{2}+\left(b-\left(L_{0}+L_{1}\right)\right) \sin q_{2}=\left(L_{3}+L_{4}+L_{5}\right) * \cos q_{3}+L_{2}$

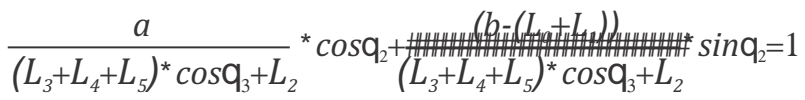

Despejando $\mathrm{q}_{2}$, se obtiene:

$\mathrm{q}_{2}=\sin ^{-}\left(\frac{z_{e}-\left(L_{0}+L_{1}\right)}{\sqrt{x_{e}^{2}+y_{e}^{2}+Z_{e}^{2}+\left(L_{0}+L_{1}\right)^{2}-2 \mathrm{~L}_{1} z_{e}}}\right) \tan -\left(\frac{\left(L_{3}+L_{4}+L_{5}\right) \sin \mathrm{q}_{3}}{L_{2}+\left(L_{3}+L_{4}+L_{5}\right) \cos \mathrm{q}_{3}}\right)$

\section{Diseño Mecánico}

Se ha empleado el software de simulación Solidworks para el diseño de los elementos del manipulador y a su vez para darle una mejor visualización (en 3D) del prototipo final mediante el renderizado de PhotoView 360 como se aprecia en la figura 3.

El diseño consta de servomotores, eslabones, sujetadores para los servos, adaptadores para servomotores, tres plataformas de soporte, un gripper, tuercas y pernos para el aseguramiento y estabilidad del manipulador al realizar diversos movimientos.

Cada servomotor representa un grado de libertad del manipulador, con la excepción del segundo grado de libertad, en el cual se ubican dos servomecanismos, esto con el objetivo de dar un mayor torque en esa zona, ya que controla mayor parte del peso del manipulador.

\section{Distribución estructural del manipulador}

Consta de una base giratoria controlada por el primer servomotor, seguido de dos servomotores en la parte superior donde empieza a enlazarse las articulaciones hasta llegar al Gripper (efector final). 


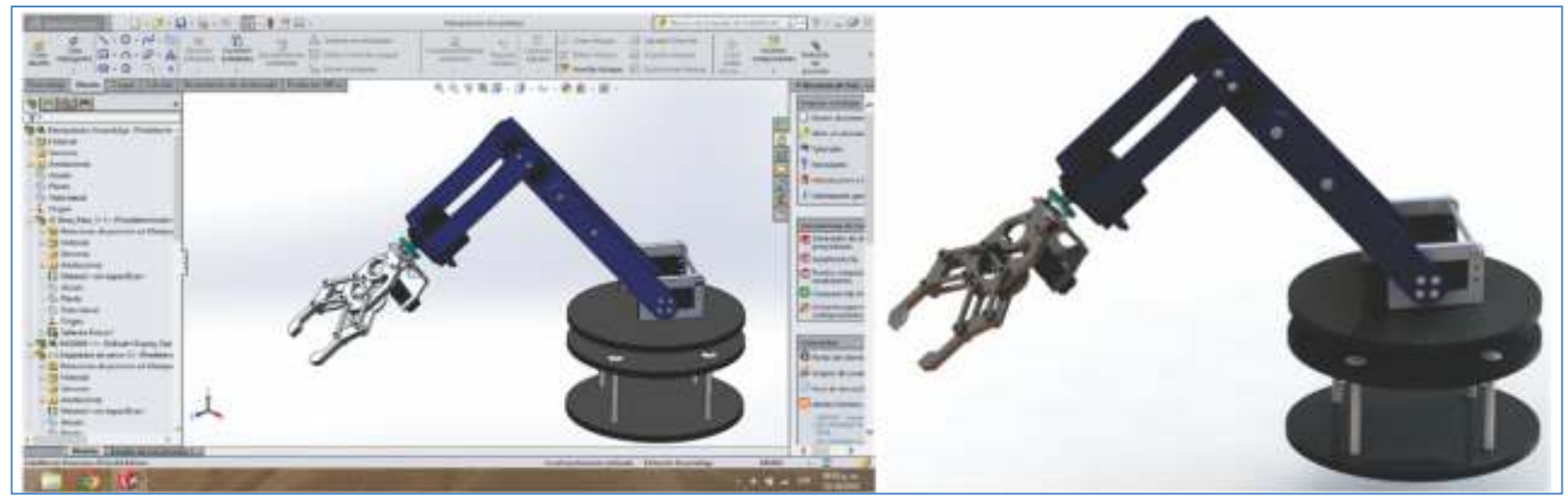

Figura 3. Diseño del Manipulador en Solidworks y Renderizado en PhotoView 360.

\section{Manufactura}

Para la elaboración de las piezas en forma real, se optó por hacer uso del sistema de corte a laser, esto con el fin de obtener una mejor precisión y material empleado para fabricación fue el acrílico de $3 \mathrm{~mm}$ y $6 \mathrm{~mm}$ de espesor.

\section{Diseño Electrónico}

Utilizando un microcontrolador $18 \mathrm{~F} 4550$ se diseñó una tarjeta para el control de la posición de los servomotores mediante la modulación por ancho de pulso (PWM). Esta tarjeta cuenta con comunicación serial mediante el protocolo RS232, usando la configuración de un MAX232 para el envío y recepción de datos.

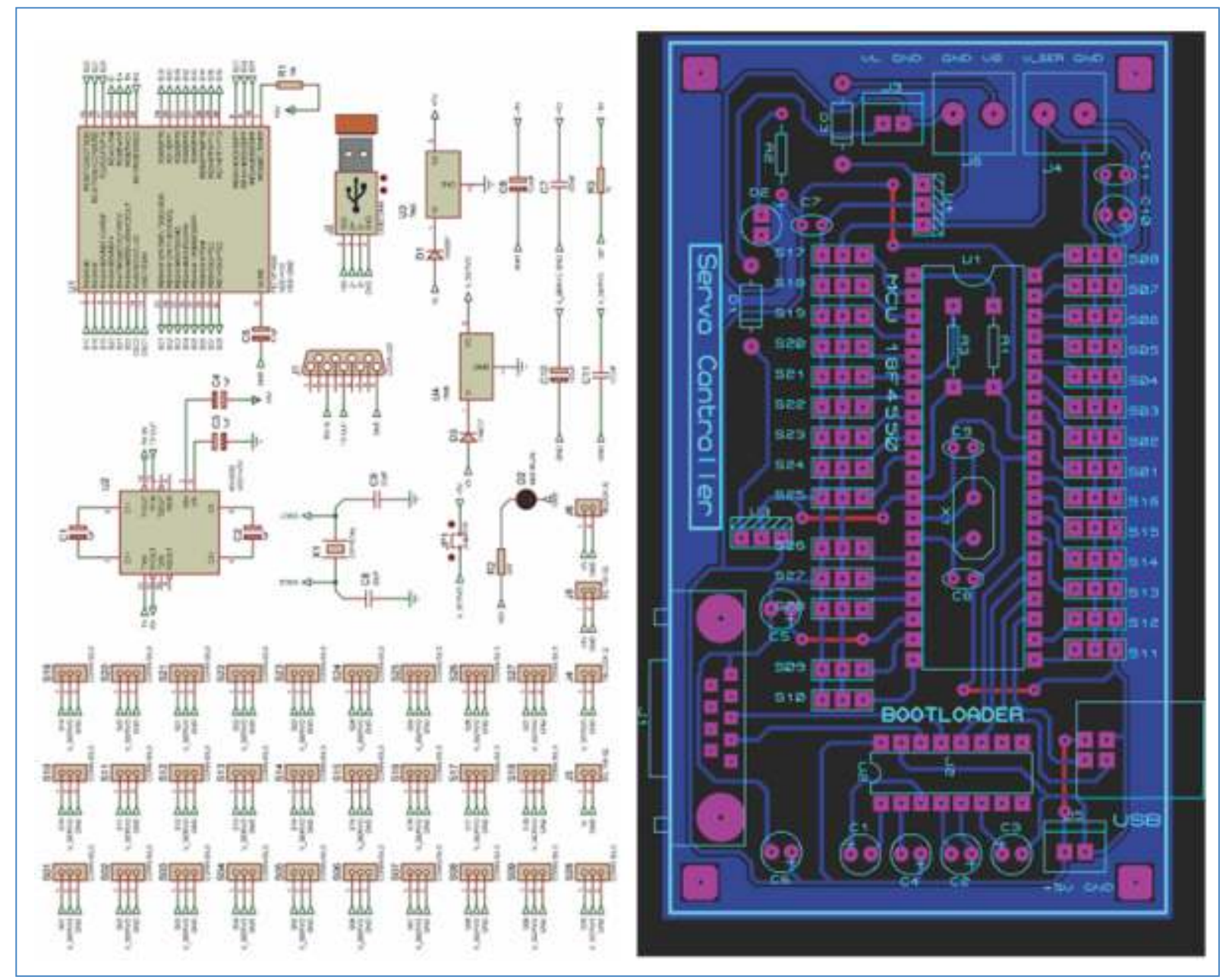

Figura 4. Esquema Diseño Electrónico y PCB Servo Controladora. 
En la siguiente imagen (Figura 5) se puede observar la simulación de la Servo controladora para 5 servomecanismos, que son controlados mediante la modulación por ancho de pulso (PWM) enviada a través del puerto serie. También se muestra el análisis de la gráfica PWM.

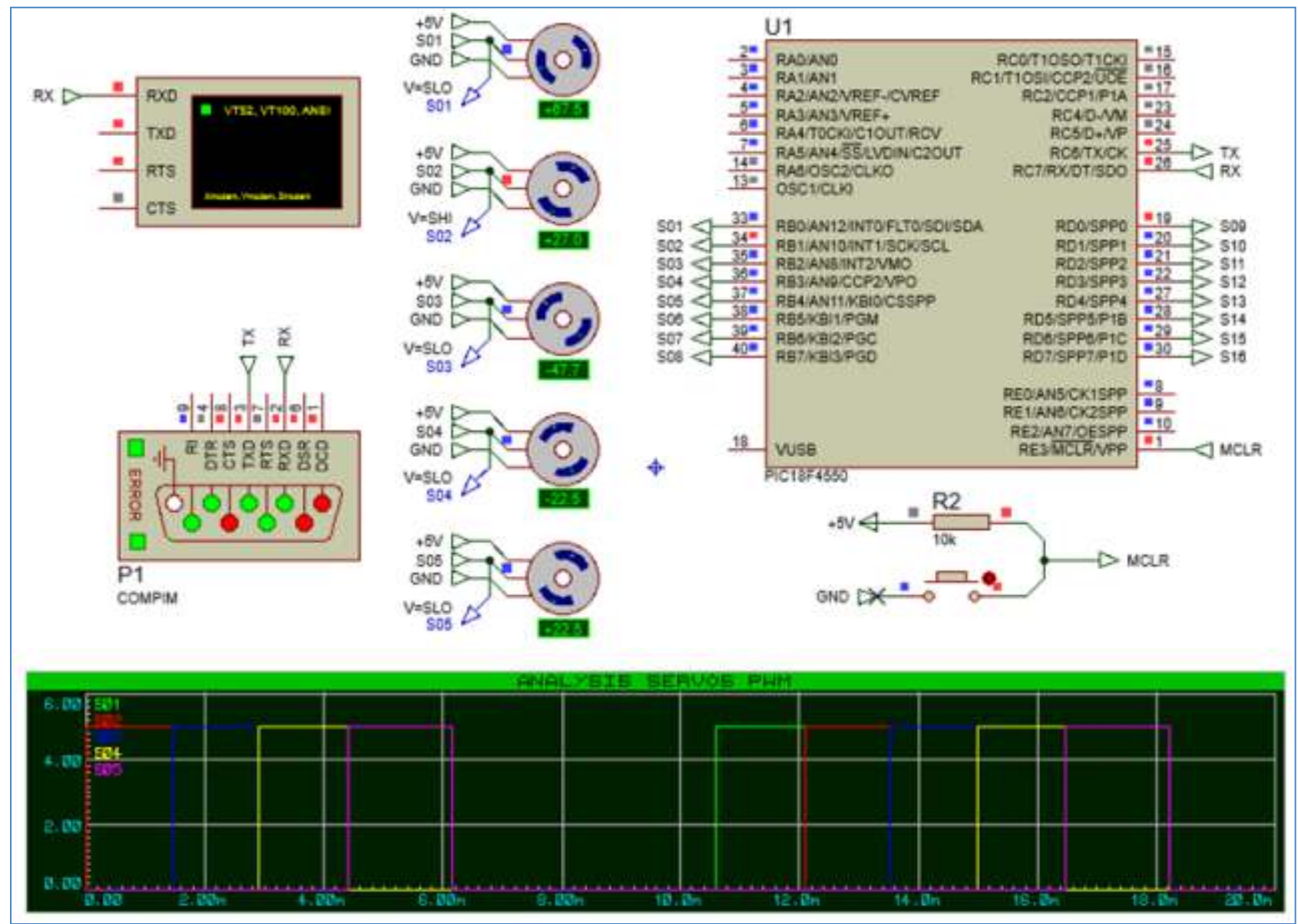

Figura 5. Simulación de la Servo Controladora.

\section{Software de Control}

El software de control interactivo se realizó en el programa LabView 2012. Esta interfaz permite controlador el manipulador robótico desde un ordenador, enviando la posición de los servomotores mediante comunicación RS232 a la tarjeta controladora conectada al manipulador. El software de control implementado también permite obtener la
Matriz de Transformada Homogénea, y realizar los cálculos de la cinemática directa y la cinemática inversa. Cuenta con una imagen en 3D de un manipulador, que nos permite visualizar la posición y orientación para la simulación previa, como también al momento de enlazarse con el manipulador, ambos realizaran el mismo movimiento tanto de forma real como virtual. 


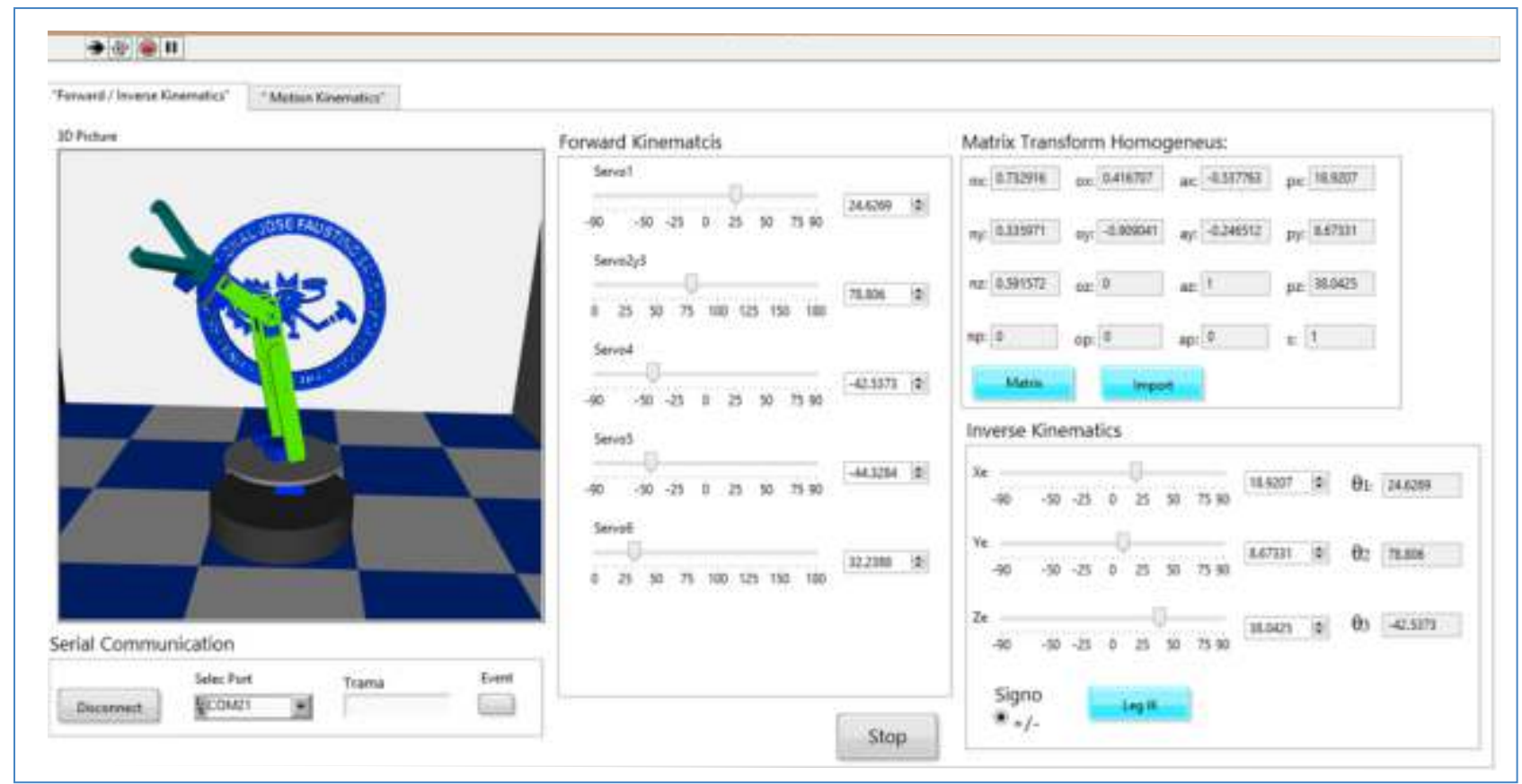

Figura 6. Interfaz del Manipulador Robótico

\section{RESULTADOS}

Se presenta los resultados obtenidos del diseño mecánico y los estudios de la cinemática directa y cinemática inversa, modelados matemáticamente mediante los parámetros de Denavit Hartenberg (DH).

\section{Diseño Mecánico del Manipulado}

El diseño mecánico implementado en su mayoría por piezas de acrílico y que fue modificado en el transcurso del desarrollo de la tesis, siendo el diseño final el que se muestra en la Figura 11.

\section{Diseño de la tarjeta controladora de servomotores}

La elaboración de la tarjeta controladora de servomotores, cuyo fin es enlazar el manipulador al ordenador, está construida en fibra de vidrio y lleva grabadas etiquetas con los nombres de los componentes que se encuentran soldados en la superficie como se muestra en la Figura 12.

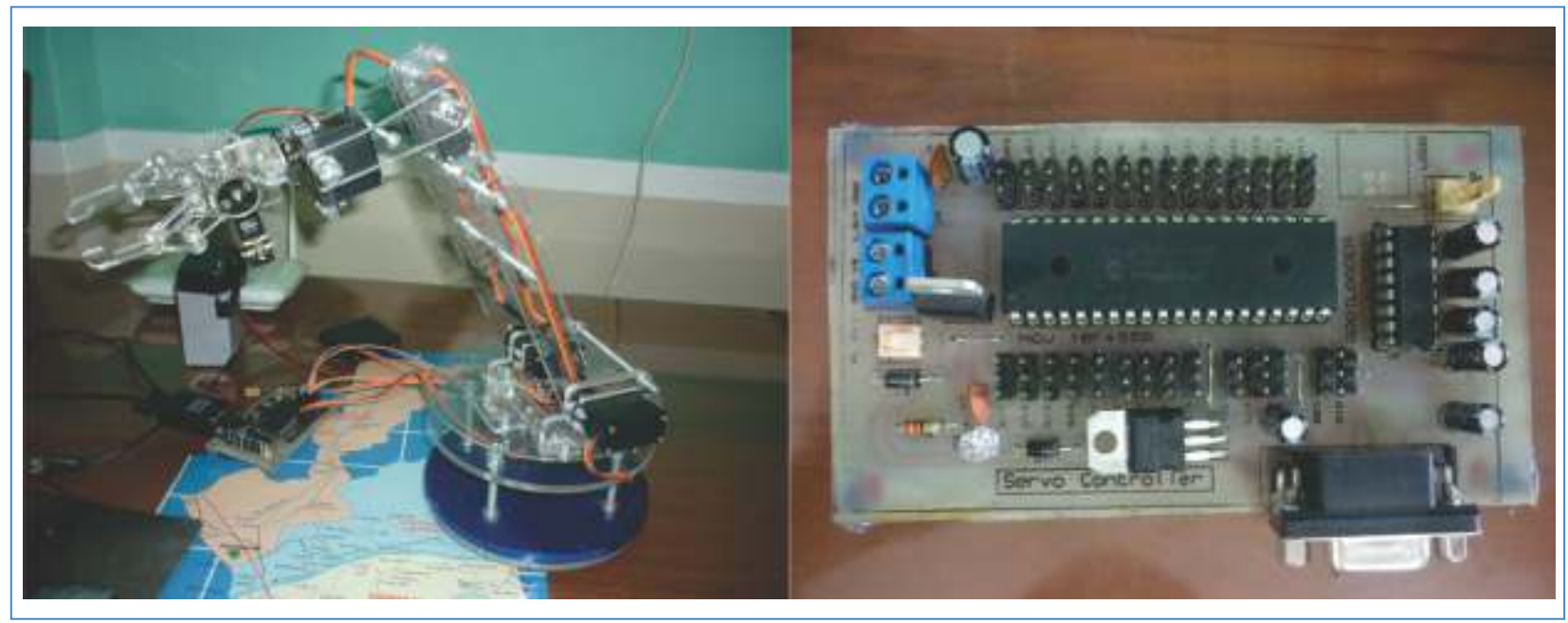

Figura 7. Robot Manipulador de cinco grados de Libertad y Tarjeta Controladora de Servomotores. 


\section{Diseño y modelado de la Interfaz Gráfica}

El diseño de la interfaz se realizó mediante el software LabView, al cual se ingresó el modelo matemático de la cinemática directa e inversa, calculados previamente mediante el software para matemática computacional Matlab, y también se incorpora una interpolación para el movimiento de una trayectoria.

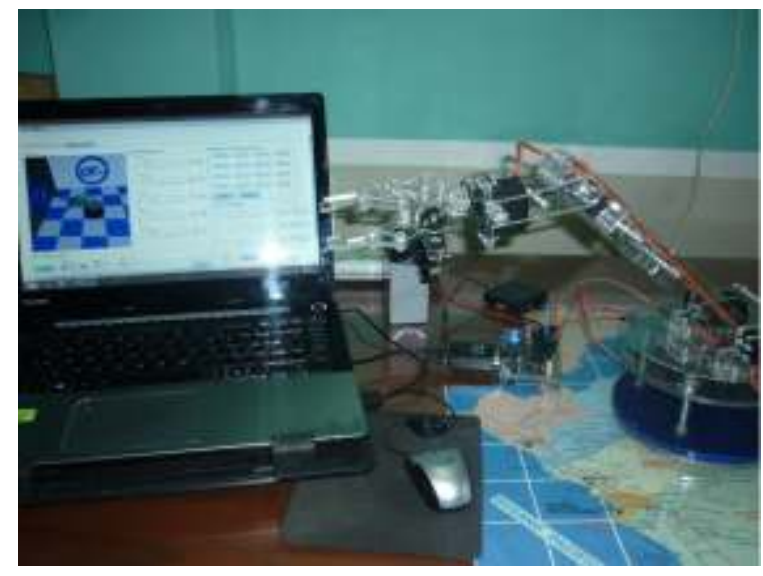

Figura 8. Interfaz de LabView conectada al robot manipulador de cinco grados de libertad.

\section{Error porcentual de la posición}

Se realizó una comparación y posterior cálculo del error entre las coordenadas del efector final $\left(x_{e}, y_{e}, z_{e}\right)$. El error porcentual de calcula mediante la siguiente formula:

$$
E P=((P C-P M) / P C) * 100 \%
$$

PC: Posición Calculada, PM: Posición Medida

Tabla 4. Obtención de las mediciones reales y el error porcentual

\begin{tabular}{|c|c|c|c|c|c|c|c|c|c|}
\hline \multirow{2}{*}{ i } & \multicolumn{3}{|c|}{ Posición Calculada } & \multicolumn{3}{|c|}{ Posición Medida } & \multicolumn{3}{|c|}{ Error Porcentual } \\
\hline & $\boldsymbol{x}_{e}$ & $y_{e}$ & $\mathbf{Z}_{e}$ & $\boldsymbol{x}_{e}$ & $y_{e}$ & $\mathbf{z}_{e}$ & $x_{e}$ & $y_{e}$ & $Z_{e}$ \\
\hline 1 & $33 \mathrm{~cm}$ & $0 \mathrm{~cm}$ & $17 \mathrm{~cm}$ & $34,5 \mathrm{~cm}$ & $0 \mathrm{~cm}$ & $17,5 \mathrm{~cm}$ & $-4,55 \%$ & $0,00 \%$ & $-2,94 \%$ \\
\hline 2 & $25 \mathrm{~cm}$ & $-10 \mathrm{~cm}$ & $20 \mathrm{~cm}$ & $25,6 \mathrm{~cm}$ & $-9,4 \mathrm{~cm}$ & $21,3 \mathrm{~cm}$ & $-2,40 \%$ & $6,00 \%$ & $-6,50 \%$ \\
\hline 3 & $10 \mathrm{~cm}$ & $30 \mathrm{~cm}$ & $20 \mathrm{~cm}$ & $10,5 \mathrm{~cm}$ & $30,7 \mathrm{~cm}$ & $19 \mathrm{~cm}$ & $-5,00 \%$ & $-2,33 \%$ & $5,00 \%$ \\
\hline 4 & $40,5 \mathrm{~cm}$ & $0 \mathrm{~cm}$ & $11,5 \mathrm{~cm}$ & $41 \mathrm{~cm}$ & $0 \mathrm{~cm}$ & $11,3 \mathrm{~cm}$ & $-1,23 \%$ & $0,00 \%$ & $1,73 \%$ \\
\hline 5 & $0 \mathrm{~cm}$ & $0 \mathrm{~cm}$ & $52 \mathrm{~cm}$ & $0 \mathrm{~cm}$ & $0 \mathrm{~cm}$ & $51,5 \mathrm{~cm}$ & $0,00 \%$ & $0,00 \%$ & $0,96 \%$ \\
\hline 6 & $0 \mathrm{~cm}$ & $30 \mathrm{~cm}$ & $20 \mathrm{~cm}$ & $0 \mathrm{~cm}$ & $30,5 \mathrm{~cm}$ & $19 \mathrm{~cm}$ & $0,00 \%$ & $-1,67 \%$ & $5,00 \%$ \\
\hline 7 & $27 \mathrm{~cm}$ & $-15 \mathrm{~cm}$ & $20 \mathrm{~cm}$ & $27 \mathrm{~cm}$ & $-15 \mathrm{~cm}$ & $19 \mathrm{~cm}$ & $0,00 \%$ & $-6,67 \%$ & $5,00 \%$ \\
\hline 8 & $40 \mathrm{~cm}$ & $0 \mathrm{~cm}$ & $38 \mathrm{~cm}$ & $39 \mathrm{~cm}$ & $0 \mathrm{~cm}$ & $38 \mathrm{~cm}$ & $2,50 \%$ & $0,00 \%$ & $0,00 \%$ \\
\hline 9 & $21 \mathrm{~cm}$ & $17,5 \mathrm{~cm}$ & $40 \mathrm{~cm}$ & $20,7 \mathrm{~cm}$ & $18 \mathrm{~cm}$ & $39 \mathrm{~cm}$ & $1,43 \%$ & $-2,85 \%$ & $2,50 \%$ \\
\hline 10 & $10 \mathrm{~cm}$ & $-26 \mathrm{~cm}$ & $20 \mathrm{~cm}$ & $10,2 \mathrm{~cm}$ & $-25 \mathrm{~cm}$ & $19 \mathrm{~cm}$ & $-2,00 \%$ & $3,85 \%$ & $5,00 \%$ \\
\hline
\end{tabular}




\section{DISCUSIÓN}

Del diseño mecánico del manipulador, se logró reducir el peso diseñando y fabricando cada parte de la estructura mecánica en acrílico de $3 \mathrm{~mm}$ y $6 \mathrm{~mm}$ de espesor a fin de conseguir una mejor estabilidad.

La tarjeta controladora de servos, que se puede apreciar en la Figura 7, tiene una capacidad para controlar hasta 20 servomecanismos de los cuales solo están siendo utilizados 6 canales, por otra parte se observa que cuenta con la implementación de un conector serial para el protocolo de comunicación Rs232.

Para el diseño y modelado de la interfaz gráfica, se incorporó el modelado matemático en LabView, mediante el mathscript para obtener la matriz de transformación homogénea, la cinemática directa, la cinemática inversa y realizar el recorrido de traslación del manipulador para el transporte de objetos.

Del error porcentual de la posición, se logró determinar que de una muestra de 10 posiciones aleatorias, existe un margen de error en su mayoría comprendidos entre $0-5 \%$, siendo algunos casos los que superan por poco el $10 \%$.

La interfaz gráfica implementada permitió realizar los cálculos matemáticos de la cinemática directa e inversa, así como también la simulación de los movimientos y trayectorias del manipulador como un estudio previo antes de realizar el prototipo de forma real.

La tarjeta controladora de servomecanismos, nos permite enlazar la simulación virtual con la real, de tal manera que se puede realizar ambos estudios en simultáneo y determinar el error porcentual de cada posición.

Por lo tanto para la construcción de un robot manipulador se deben tener ciertos criterios como:

Los eslabones y articulaciones del manipulador, estos deben ser de un material rígido pero que a su vez no presente demasiado peso para evitar sobrecargar la capacidad de torque de los servomotores.
Diseñar un sistema estable, para evitar las vibraciones y posteriores mediciones erróneas de la posición y fijar una base sólida para el manipulador, con el fin de evitar que se balancee en las trayectorias.

Por otra parte queda demostrado lo planteado por Barrientos et al. (1997) y Craig (2006), quienes proponen que la utilización de los Parámetros de Denavit Hartenberg nos permite diseñar y modelar una plataforma robótica.

Podemos concluir que el manipulador robótico de cinco grados de libertad cumplió con los objetivos del diseño, modelamiento y la implementación, siendo este de material (acrílico) liviano y resistente, con excepción de la base la cual es de plomo con el fin de tener una estabilidad al momento de realizar las trayectorias.

Finalmente sabemos que hoy en día se necesita que los estudiantes realicen más investigación y para ello deben contar con herramientas que les permitan llevarlo a la práctica. Se espera que el trabajo de investigación presentado contribuya y sirva como modelo para que cualquier estudiante pueda construir su propio manipulador robótico, y programarlo para realizar diversos tipos de investigaciones relacionadas a esta área como pueden ser la cinemática directa y la cinemática inversa.

\section{AGRADECIMIENTO}

Al Ing. Guillermo Evangelista Adrianzén, gracias por haberme brindado los conocimientos necesarios en la etapa de pre grado que fueron de valiosa información para elaborar la presente investigación.

\section{REFERENCIAS BIBLIOGRÁFICAS}

Barrientos, A., Peñín, L., Balaguer, C. \& Aracil, R. (1997). Fundamentos de la Robótica (1era Ed.). Madrid: McGraw-Hill Interamericana.

Bolton, W. (2001). Mecatrónica (2da Ed.). México D.F.:Alfaomega.

Craig, J. (2006.) Robótica (3era Ed.). México D.F., México: Prentice Hall. 
Kelly, R. \& Santibañez, V. (2003) Control de Movimiento de Robots Manipuladores (1era Ed.) México: Prentice Hall.

Subir, K. (2008). Introducción a la Robótica. México D.F.: M c Graw-Hill Interamericana.
Correo Electrónico: ediazronceros@gmail.com

Revisión de pares:

Recibido: 22-04-2016

Aceptado: 22-06-2016 